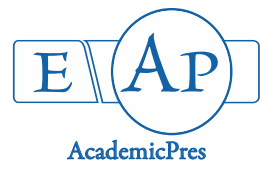

\title{
Chemical Composition, Toxicity and Antifungal Activities of Megaphrynium macrostachyum (K. Schum) Leaf Extract Against Foodborne Fungi
}

\section{Oluwagbenga Oluwasola ADEOGUN ${ }^{1 *}$, Adedotun Adeyinka ADEKUNLE ${ }^{1}$, Erute Magdalene ADONGBEDE ${ }^{1}$, Tom Anofi ASHAFA ${ }^{2}$}

\author{
${ }^{1}$ University of Lagos, Faculty of Science, Department of Botany, Akoka, Lagos State, Nigeria; aaded63@yahoo.com; erute70@yahoo.com; \\ adeogunoluwagbenga@gmail.com (*orrespondingauthor) \\ ${ }^{2}$ University of Free State, Department of Plant Sciences, Qwa Qwa Campus, Free State, South Africa; tom.ashafa@gmail.com
}

\begin{abstract}
This study aimed to examine the preservative potential of Megaphrynium macrostachyum on fungi responsible for the deterioration of orange juice and corn Jell-O. The phytochemicals from plants' leaves were extracted with four solvents: acetone, aqueous, ethanol and hexane. The solvents were differently and tested against fungi isolated from orange juice and corn Jell-O using disc diffusion method. Phytochemical screening of the extracts from the leaves was carried out, and the most active extract was tested via GC-MS for the essential oils and HPLC fingerprinting. The toxicity test of the extracts against brine shrimp was carried out after exposure for 24 hours. The toxicity test showed that the extracts were non-toxic on the Brine Shrimps at $\mathrm{LC}_{50}(379.21 \mu \mathrm{g} / \mathrm{ml}$ and $107.21 \mu \mathrm{g} / \mathrm{ml}$ for aqueous and ethanol extracts). The qualitative phytochemical test reported the presence of alkaloids, tannins, saponins, flavonoids, steroids and terpenoids in different extracts of the plant' leaves. The quantitative phytochemical determination of the most active extract revealed alkaloids with the highest contents of $107.48 \mathrm{mg} / 100 \mathrm{~g}$. The GC-MS analyses of the fresh leaves of the plants revealed the presence of isodecane with the highest percentage at $15.56 \%$. The GC-MS analyses of the dried leaves revealed isodecane with the highest percentage at $10.43 \%$. The HPLC analysis revealed the presence of various phytochemical constituents in the dried leaves. This study has been able to establish the potency of Megaphrynium macrostachyum leaves on fungi associated with the spoilage of Citrus sinensis (orange) juice and Corn Jell-O ('Eko') which contribute to tremendous research towards the use and acknowledgment of natural antimicrobials for the preservation of food.
\end{abstract}

Keywords: Citrus sinensis juice, corn Jell-o, extracts, food spoilage, lethality assay, M. macrostachyum

\section{Introduction}

The growing improvement in food production techniques has not been able to address the low yield of food crop due to postharvest losses, which arise due to handling and microbial infection (Nedorosteva et al., 2008). In developing countries, postharvest losses can be severe due to inadequate storage and transportation facilities (Al-Hindi et al., 2011). Food safety and wastage are of elemental concern to the consumers. Food industries now use synthetic preservatives to prevent the growth of foodborne microbes, it has been suggested that some of these synthetic preservatives convert some ingested materials into toxic substances or carcinogens by increasing the activity of microsomal enzymes (Skocibusic et al., 2006). Consumer apprehension over the use of synthetic preservatives has resulted in an increased interest in natural antimicrobial alternatives to enhance the shelf life and safety of food products. It has been established that most plants produce antimicrobial metabolites, either as part of their normal program of growth and development or in response to pathogens attack or stress (Skocibusic et al., 2006; Cock and Van Viuren, 2015). The use of natural antimicrobials has been receiving a great share of consideration for the control of microorganisms responsible for spoilage of food materials. They have extensively been used to control microbial contamination of food, improving shelf life extension technologies to eliminate undesirable pathogens or delay microbial spoilage and development of antibiotic resistance by microorganisms or strengthening immune cells in humans (Tajkarimi et al., 2010). 
362

Edible plants and plant products have been used in foods since antiquity as preservative agents as well in folk medicines due to their antimicrobial activities. The inhibitory properties of many edible plant extracts against bacteria and fungi associated with food spoilage have been explored by several researchers (Skocibusic et al., 2006; Tajkarimi et al., 2010; Cock and Van Vuuren, 2015).

Researches on plants for the preservative purpose are exponentially growing all over the world because of consumers yearning for high quality and safe food with extended shelf life. In Sub-Saharan Africa with fewer technologies and lack of access to refrigerating equipment, the natives employ the use of leaves to wrap food in order to enhance its shelf-life. Plant leaves of Musa paradisiaca, Cola nitida, Theobroma cacao, Colocasia esculenta, Canna indica, Megaphrynium macrostachyum, Thaumatococcus daniellii, etc. (Adegunloye et al., 2006) are used in packaging and presenting foods to clientele. The leaf extracts of these plants have been documented to possess antimicrobial properties (Adegunloye et al., 2006; Erturk, 2006; Yazdani et al., 2011) and essential oils from plants have been generally recognized as being safe and while the toxic impact of these plants on food might be quite inconsequential (Borbosa et al., 2009; Mith et al., 2014).

Megaphrynium macrostachyum (K.Schum.) (Family: Marantaceae) is found in the rainforest of the west and central Africa (Milne-Redhead, 1952). It is a pubescent herb with rhizome up to $6 \mathrm{~m}$ and it has long bearing singly numerous leaves (Milne-Redhead, 1952). The leaf is papery and is much used as a roof-thatch. They are used to wrap foodstuffs, tapioca roots, kolanut, meat, etc., and as a packing material (Milne-Redhead, 1952).

This study aimed to determine the in-vitro antifungal potential of organic extracts on foodborne fungi isolated from corn Jell-O and fruit juices and also determine the invivo lethality status on brine shrimps for toxicity appraisal of the leaf extracts.

\section{Materials and Methods}

\section{Plantmaterial}

Megaphrynium macrostachyum leaves were collected from Obere in Otta local government area of Ogun state (Nigeria, N 06 $40.695^{\prime}$, E 003.06 .385 ). The plant was identified and deposited at Lagos University Herbarium (LUH) for repository purpose with voucher no: Lagos University Herbarium (LUH) 6088.

\section{Sources offungi}

The fungi used for this study were isolated from orange juice and corn Jell-O (a Nigerian Jell-O). The conventional method of isolation was adopted (Alwakeel, 2013; Samuel and Orji, 2015); they were identified by comparing their morphology with fungi descriptions of Bryce (1992) and Beveridge (2001) and sent to CABI, Surrey, Egham for molecular Identification and confirmation.

\section{Preparation of inocula}

The fungi for anti-fungal assay were obtained via pure culture plates. The spore suspensions of the fungi were made using sterilized normal saline water of $10 \mathrm{ml}$ and the suspensions were observed under the microscope and later counted using hemocytometer (Adekunle and Ikumapayi, 2006) and then further diluted to approximately $10^{6}$ CFU/mL (Kosanic et al., 2012).

\section{Preparation of plant organic extracts}

The leaves of $M$. macrostachyum were shade-dried for ten days and were ground using a miller. Two hundred grams of the leaves were extracted with four different solvents: aqueous, acetone, ethanol and hexane; the method of extraction follows what was described by Silva et al. (2014).The yields of the extracts were determined using the method of Dellavalle et al. (2010) with slight modification.

Each extract obtained was tested to ensure its purity via dropping on sterile plates containing potato dextrose agar and well spread on the agar. The plates were incubated and examined for possible growth of contaminants, the absence of contaminants confirmed the purity of the test extracts (Okigbo et al., 2009).

\section{The antifungal assay}

The discs for anti-fungal activity were made from filter papers (Whatman No. 1 filter paper) and the methods used were based on methods adopted by Adekunle and Ikumapayi (2006) and Valle et al. (2016).

The concentrations of plant extracts were determined after dryness of the plant extracts using the method of Zakia et al. (2015). The concentrated extracts were reconstituted with $100 \mathrm{ml}$ of Dimethyl sulfoxide (DMSO) and the extracts were later varied into different concentrations: $0.1 \mathrm{~g}$ $(10 \mathrm{mg} / \mathrm{ml}), 0.5 \mathrm{~g}(50 \mathrm{mg} / \mathrm{ml}), 1 \mathrm{~g}(100 \mathrm{mg} / \mathrm{ml}), 5 \mathrm{~g}(500$ $\mathrm{mg} / \mathrm{ml})$ and $10 \mathrm{~g}(1,000 \mathrm{mg} / \mathrm{ml})$.

\section{Determination of antifungal assay of plant extracts against isolated fungi}

The disc diffusion method adopted by Adekunle and Ikumapayi (2006) and Zakia et al. (2015) was adopted for the antifungal assay. The tests were conducted with authenticated pure cultures of the fungi by aseptically dropping $0.5 \mathrm{ml}$ of the stocked suspension of each fungus over the entire agar surface of freshly prepared Potato Dextrose Agar (PDA) plates and spread evenly with the aid of a spreader. The discs (4) impregnated with the extracts were placed at the four edges of the surface of the agar plate with the aid of a sterilized forceps. The discs were also impregnated with Dimethyl sulfoxide (DMSO) as negative control and Nystatin (antifungal drug) as a positive control.

\section{(MIC)}

Determination of Minimum Inbibitory Concentration

Minimum inhibitory concentration (MIC) was determined for each plant extract showing antimicrobial activity. Modified methods of Ngonda et al. (2012) and Mishra et al. (2015) were adopted. Concentrations of the extracts with no observable fungal growth were taken as the minimum inhibitory concentration. 
Determination of Minimum Fungicidal Concentration $(M F C)$

Minimum fungicidal concentration (MFC) was determined for each plant extract showing antimicrobial activity. A Modified method of Ngonda et al. (2012), Mihai and Popa (2015) and Oro et al. (2015) were adopted; Petri dishes of the concentrations of extracts that showed no visible fungal growth were seeded into freshly prepared PDA plate to assay for the fungicidal effect of the extracts. The plates containing the test organisms were incubated at $28^{\circ} \mathrm{C}$. The minimum fungicidal concentration was regarded as the lowest concentration that did not yield any fungal growth on the solid medium used.

\section{Lethality test using brine shrimp}

The brine shrimps lethality test using the larvae of brine shrimp nauplii (Artemia salina L.) was carried out using the method described by Ashafa et al. (2013) and Kayode and Afolayan (2015), with slight modification. $1 \mathrm{mgml}^{-1}$ of the extract was prepared from the original stock solution of $M$. macrostachyum that was used for antifungal assays. The extracts were further diluted to final concentrations of 20, 40, 60, 80 and $100 \mathrm{ugml}^{-1}$ in different vials using DMSO.

\section{Qualitative and quantitative phytochemical screening}

The acetone, aqueous, hexane and ethanol extracts of $M$. macrostachyum leaves were subjected to qualitative preliminary phytochemical screening by adopting standard methods as described by Harborne (1973), Trease and Evans (1989), Sofowora (1993), Edeoga et al. (2005) and Ashafa and Umebese (2012). They were later subjected to quantitative analysis of the phytochemical constituents based on methods of Edeoga et al. (2005) and Prohp and Onoagbe (2012).

\section{Extraction and analysis of essential oils}

The essential oils from fresh and dried leaves of $M$. macrostachyum were extracted and analyzed based on the method described by Ashafa, Grierson and Afolayan (2008).

\section{Determination of phytoconstituents by high-performance liquid chromatography \\ The crude extracts of ethanol which showed good inhibitory activities on all test organisms were analyzed for the presence of phytoconstituents through HPLC using a method employed by Ngonda et al. (2012).}

\section{Statistical analysis}

The data obtained were analyzed using analysis of variance (ANOVA) and compared using Duncan's multiple range test (DMRT) (Leech et al., 2008).

\section{Results}

The isolated test fungi were Aspergillus aculeatus, Aspergillus niger, Aspergillus flavus, Rhizopus stolonifer, Issatchenkia orientalis, Meyerozyma guilliermondii, Fusarium oxysporium, Paecilomyces variotti, Penicillium crustosum, Trichoderma harzianum and Meyerozyma caribbica.

The yield of the extracts is depicted in Table 1; it was observed that the aqueous extract had the highest yield among the solvents used for extraction.

The observable activity of the extract on agar medium as depicted in plate 1 showed that the extract had not been contaminated before use.

Antifungal Assays of $M$. macrostachyum on fungi isolated from orange juice and corn Jell-O

The concentration of acetone, ethanol, hexane and aqueous extracts from $M$. macrostachyum increased with an increase in diameter of the zone of inhibition as described in Table 2. Analysis of variance revealed that the rate of inhibition differs significantly $(\mathrm{P}<0.05)$ along the varied concentrations of the extracts against each test isolate. The concentrations of ethanol extracts against some of the test fungi such as Issatchenkia orientalis increased from $11.7 \pm 0.7$ $\mathrm{mm}$ at $0.1 \mathrm{~g}$ to $13.0 \pm 0$ at $10 \mathrm{~g}$, Meyerozyma guilliermondii increased from $11.3 \pm 0.3 \mathrm{~mm}$ at $0.1 \mathrm{~g}$ to $13.3 \pm 0 \mathrm{~mm}$ at $10 \mathrm{~g}$ while Meyerozyma caribbica increased from $10.7 \pm 0.3 \mathrm{~mm}$ at $0.1 \mathrm{~g}$ to $14.0 \pm 0 \mathrm{~mm}$ at $10 \mathrm{~g}$ and the increment is also applicable to other test fungi with different extracts of $M$. macrostachyum leaves. The activity of the antifungal drug (Nystatin) on test fungi is less active compared to leaf extracts. Ethanol extract was more pronounced with higher activities across all tested fungi compared with the activities of aqueous, acetone and hexane extracts (Table 2). It was observed based on table 3 that the ethanol extract had the lowest minimum inhibitory concentration among the test extracts on test fungi, the range of the MIC of ethanol extract is between the range $0.5 \mathrm{~g}$ to $10 \mathrm{~g}$ (Table 3 ). The minimum fungicidal concentration of extracts of leaf of $M$. macrostachyum exhibited the potency to kill the test fungi at a range of $0.1 \mathrm{~g}$ to $10.0 \mathrm{~g}$ (Table 3 ) and it was also manifested that the rate of the fungicidal activities of ethanol was higher than other test extracts of $M$. macrostachyum leaves with values that range between $0.1 \mathrm{~g}$ to $10 \mathrm{~g}$ on most of the test fungi.

Brine shrimps lethality assay after 24 hours of exposure

The lethality concentration of extracts required to kill fifty percent of the population of Brine Shrimps is depicted in Table 4. The ethanol and aqueous extracts of $M$. macrostachyum of $107.21 \mu \mathrm{g} / \mathrm{ml}$ and $379.21 \mu \mathrm{g} / \mathrm{ml}$ are nontoxic because they were above the general toxicity test agreement of $\mathrm{LC}_{50}$ being above $100 \mathrm{\mu g} / \mathrm{ml}$ for toxic-free substances.

Table 1. Percentage yield of $M$. macrostachyum leaf extract

\begin{tabular}{cccc}
\hline Solvent & Yield of extract $(\mathrm{g})$ & Yield of extract $(\%)$ & 6.06 \\
\hline Acetone & 12.1 & 13.4 \\
Ethanol & 26.8 & 12.4 \\
Hexane & 24.2 & 33.5 \\
Aqueous & 67 & 3 \\
\hline
\end{tabular}


Table 2. Concentration and zones of inhibition of Megaphrynium macrostachyum leaf extracts against the test fungi

\begin{tabular}{|c|c|c|c|c|c|c|c|c|c|c|c|c|}
\hline Extract & conc & ANS & AN & AFS & MCR & YST & YSTA & YSTB & FSR & YB & PENU & TRI \\
\hline \multirow{5}{*}{ Acetone } & 0.1 & $8 \pm 0^{\mathrm{b}}$ & $7.7 \pm 0.3^{b}$ & $7 \pm 0^{b}$ & $5 \pm 0^{a}$ & $9.3 \pm 0.3^{c}$ & $9 \pm 0.6^{\mathrm{bc}}$ & $9 \pm 0^{c}$ & $6 \pm 0^{2}$ & $9 \pm 0^{b}$ & $9 \pm 0^{b}$ & $9.7 \pm 0.3^{c}$ \\
\hline & 0.5 & $9.3 \pm 0.3^{c}$ & $8.7 \pm 0.3^{c}$ & $9.7 \pm 0.7^{\mathrm{d}}$ & $6 \pm 0^{\mathrm{b}}$ & $9.7 \pm 0.3^{c}$ & $9.3 \pm 0.3^{\mathrm{cd}}$ & $9.3 \pm 0.3^{c}$ & $6 \pm 0^{2}$ & $9.3 \pm 0.3^{b}$ & $9.3 \pm 0.3^{b}$ & $11 \pm 0^{\mathrm{d}}$ \\
\hline & 1.0 & $10.7 \pm 0.3^{d}$ & $9 \pm 0^{c}$ & $9 \pm 0^{d}$ & $6 \pm 0^{b}$ & $9.7 \pm 0.3^{c}$ & $10.3 \pm 0.3^{\mathrm{d}}$ & $11 \pm 0^{\mathrm{d}}$ & $6.7 \pm 0.3^{a}$ & $9.3 \pm 0.3^{\mathrm{b}}$ & $9.3 \pm 0.3^{b}$ & $11 \pm 0^{d}$ \\
\hline & 5.0 & $11 \pm 0^{\mathrm{d}}$ & $10.7 \pm 0.3^{\mathrm{d}}$ & $9.3 \pm 0.3^{d}$ & $10 \pm 0^{\mathrm{d}}$ & $11.3 \pm 0.3^{\mathrm{d}}$ & $12.3 \pm 0.3^{e}$ & $11.7 \pm 0^{\mathrm{d}}$ & $9.3 \pm 0.3^{\mathrm{d}}$ & $12.3 \pm 0.7^{\mathrm{c}}$ & $11.7 \pm 1.2^{c}$ & $11 \pm 0.6^{c}$ \\
\hline & 10.0 & $12 \pm 0^{c}$ & $11.3 \pm 0.3^{\mathrm{d}}$ & $11.3 \pm 0.3^{e}$ & $11 \pm 0^{\mathrm{d}}$ & $15 \pm 0.6^{c}$ & $16 \pm 0.6^{f}$ & $12 \pm 0.6^{\mathrm{e}}$ & $10 \pm 0.6^{\mathrm{d}}$ & $13.3 \pm 0.3^{c}$ & $12.7 \pm 0.9^{c}$ & $12.7 \pm 0.7^{\mathrm{e}}$ \\
\hline \multirow[t]{2}{*}{ Nystatin } & $\mathrm{PO}$ & $8 \pm 0^{\mathrm{b}}$ & $7.3 \pm 0.3^{b}$ & $8 \pm 0^{c}$ & $8 \pm 0^{c}$ & $7.3 \pm 0.3^{b}$ & $8 \pm 0^{\mathrm{b}}$ & $7.7 \pm 0.3^{\mathrm{b}}$ & $8 \pm 0^{c}$ & $8.3 \pm 0.3^{b}$ & $8 \pm 0^{\mathrm{b}}$ & $7.7 \pm 0.3^{b}$ \\
\hline & 0.1 & $5 \pm 0^{a}$ & $5 \pm 0^{a}$ & $5 \pm 0^{\mathrm{a}}$ & $5 \pm 0^{\mathrm{a}}$ & $9.3 \pm 0.3^{c}$ & $5 \pm 0^{2}$ & $5 \pm 0^{a}$ & $5 \pm 0^{a}$ & $5 \pm 0^{a}$ & $5 \pm 0^{2}$ & $5 \pm 0^{\mathrm{a}}$ \\
\hline \multirow{4}{*}{ Hexane } & 0.5 & $5 \pm 0^{a}$ & $5 \pm 0^{x}$ & $5 \pm 0^{a}$ & $5 \pm 0^{a}$ & $9.7 \pm 0.3^{c}$ & $5 \pm 0^{a}$ & $7.3 \pm 0.3^{b}$ & $5 \pm 0^{2}$ & $6 \pm 0^{b}$ & $5 \pm 0^{a}$ & $5 \pm 0^{2}$ \\
\hline & 1.0 & $5 \pm 0^{a}$ & $5 \pm 0^{2}$ & $5 \pm 0^{\mathrm{a}}$ & $5 \pm 0^{a}$ & $9.7 \pm 0.3^{c}$ & $6.3 \pm 0.3^{b}$ & $8 \pm 0^{\mathrm{bc}}$ & $6.3 \pm 0.3^{\mathrm{b}}$ & $7 \pm 0^{\mathrm{cd}}$ & $5 \pm 0^{2}$ & $5 \pm 0^{a}$ \\
\hline & 5.0 & $8.7 \pm 0.3^{c}$ & $7.3 \pm 0.3^{b}$ & $8 \pm 0^{a}$ & $5 \pm 0^{a}$ & $11.3 \pm 0.3^{\mathrm{d}}$ & $6.7 \pm 0.3^{\mathrm{bc}}$ & $8.3 \pm 0.3^{c}$ & $7.3 \pm 0.3^{b c}$ & $6.7 \pm 0.3^{\mathrm{bc}}$ & $6.7 \pm 0.3^{b}$ & $5 \pm 0^{a}$ \\
\hline & 10.0 & $9 \pm 0^{c}$ & $7 \pm 0^{\mathrm{b}}$ & $8.7 \pm 0.3^{c}$ & $7 \pm 0.6^{b}$ & $15 \pm 0.6 \mathrm{e}$ & $7 \pm 0^{c}$ & $9 \pm 0^{d}$ & $7.3 \pm 0.9^{\mathrm{bc}}$ & $7.7 \pm 0.7^{\mathrm{de}}$ & $7 \pm 0^{\mathrm{b}}$ & $8 \pm 2^{b}$ \\
\hline \multirow[t]{2}{*}{ Nystatin } & PO & $8 \pm 0^{b}$ & $7.3 \pm 0.3^{\mathrm{b}}$ & $8 \pm 0^{\mathrm{b}}$ & $8 \pm 0^{c}$ & $7.3 \pm 0.3^{\mathrm{b}}$ & $8 \pm 0^{b}$ & $7.7 \pm 0.3^{\mathrm{b}}$ & $8 \pm 0^{c}$ & $8.3 \pm 0.3^{e}$ & $8 \pm 0^{\mathrm{b}}$ & $7.7 \pm 0.3^{\mathrm{b}}$ \\
\hline & 0.1 & $9 \pm 0^{c}$ & $9.3 \pm 0.3^{c}$ & $9 \pm 0^{\mathrm{bc}}$ & $9 \pm 0^{c}$ & $11.7 \pm 0.7^{c}$ & $11.3 \pm 0.3^{c}$ & $10.7 \pm 0.3^{c}$ & $8.7 \pm 0.3^{\mathrm{bc}}$ & $9.3 \pm 0.3^{b}$ & $9 \pm 0.6^{\mathrm{bc}}$ & $9.3 \pm 0.3^{\mathrm{bc}}$ \\
\hline \multirow{4}{*}{ Ethanol } & 0.5 & $10.3 \pm 0.3^{\mathrm{d}}$ & $11 \pm 0^{\mathrm{d}}$ & $10 \pm 0.6^{c}$ & $9.3 \pm 0.3^{\mathrm{cd}}$ & $12 \pm 0^{\mathrm{cd}}$ & $12.3 \pm 0.3^{\mathrm{d}}$ & $11 \pm 0^{c}$ & $9.3 \pm 0.3^{\mathrm{cd}}$ & $9.3 \pm 0.3^{b}$ & $9.7 \pm 0.7^{\mathrm{cdd}}$ & $10 \pm 0^{c}$ \\
\hline & 1.0 & $11 \pm 0^{c}$ & $11.3 \pm 0.3^{d}$ & $11.3 \pm 0.3^{d}$ & $10.7 \pm 0.3^{\mathrm{cdd}}$ & $12 \pm 0.6^{c, d}$ & $13 \pm 0^{\mathrm{d}, e}$ & $13 \pm 0^{d}$ & $9.7 \pm 0.3^{d}$ & $11.3 \pm 0.3^{c}$ & $10.3 \pm 0.3^{\mathrm{d}, e}$ & $10.3 \pm 0.3^{c}$ \\
\hline & 5.0 & $11.7 \pm 0.3^{f}$ & $11.7 \pm 0.3^{\mathrm{d}, e}$ & $11.7 \pm 0.3^{d}$ & $11 \pm 1.2^{\mathrm{d}}$ & $12.7 \pm 0.3^{\mathrm{cd} d}$ & $13.3 \pm 0.3^{e}$ & $13.7 \pm 0.3^{e}$ & $10 \pm 0.6^{\mathrm{d}}$ & $11.7 \pm 0^{c}$ & $11 \pm 0^{e}$ & $10 \pm 0^{c}$ \\
\hline & 10.0 & $12.3 \pm 0.3^{8}$ & $12.3 \pm 0.3^{\mathrm{e}}$ & $12.3 \pm 0.7^{d}$ & $13.3 \pm 0.3^{e}$ & $13 \pm 0^{\mathrm{d}}$ & $13.3 \pm 0.3^{e}$ & $14 \pm 0^{e}$ & $12 \pm 0^{\mathrm{e}}$ & $12 \pm 0.7^{c}$ & $11.3 \pm 0.3^{e}$ & $10 \pm 1.5^{c}$ \\
\hline \multirow[t]{3}{*}{ Nystatin } & $\mathrm{PO}$ & $8 \pm 0^{\mathrm{b}}$ & $7.3 \pm 0.3^{a}$ & $8 \pm 0^{\mathrm{b}}$ & $8 \pm 0^{\mathrm{b}}$ & $7.3 \pm 0.3^{\mathrm{a}}$ & $8 \pm 0^{\mathrm{b}}$ & $7.7 \pm 0.3^{\mathrm{b}}$ & $8 \pm 0^{\mathrm{b}}$ & $8.3 \pm 0.3^{\mathrm{b}}$ & $8 \pm 0^{\mathrm{b}}$ & $7.7 \pm 0.3^{\mathrm{b}}$ \\
\hline & 0.1 & $5 \pm 0^{a}$ & $5 \pm 0^{a}$ & $5 \pm 0^{a}$ & $5 \pm 0^{a}$ & $5 \pm 0^{2}$ & $5 \pm 0^{a}$ & $5 \pm 0^{a}$ & $5 \pm 0^{a}$ & $5 \pm 0^{\mathrm{a}}$ & $5 \pm 0^{2}$ & $13 \pm 0.9^{d}$ \\
\hline & 0.5 & $5 \pm 0^{\mathrm{a}}$ & $5 \pm 0^{2}$ & $6.3 \pm 0.3^{b}$ & $5 \pm 0^{a}$ & $5 \pm 0^{a}$ & $5 \pm 0^{a}$ & $5 \pm 0^{a}$ & $5 \pm 0^{2}$ & $5 \pm 0^{a}$ & $5 \pm 0^{a}$ & $5 \pm 0^{\mathrm{a}}$ \\
\hline \multirow[t]{3}{*}{ Water } & 1.0 & $5 \pm 0^{a}$ & $5 \pm 0^{a}$ & $7 \pm 0^{c}$ & $5 \pm 0^{a}$ & $5 \pm 0^{2}$ & $5 \pm 0^{a}$ & $5 \pm 0^{a}$ & $5 \pm 0^{a}$ & $5 \pm 0^{2}$ & $5 \pm 0^{a}$ & $13 \pm 0.6^{d}$ \\
\hline & 5.0 & $6.3 \pm 0.3^{b}$ & $5 \pm 0^{a}$ & $7 \pm 0^{c}$ & $5 \pm 0^{a}$ & $5 \pm 0^{\mathrm{a}}$ & $5 \pm 0^{a}$ & $5 \pm 0^{2}$ & $5 \pm 0^{a}$ & $5 \pm 0^{a}$ & $5 \pm 0^{a}$ & $9.7 \pm 0.9^{c}$ \\
\hline & 10.0 & $10.3 \pm 0.3^{d}$ & $10.3 \pm 0.3^{c}$ & $7.3 \pm 0.3^{c}$ & $8.3 \pm 0.7^{b}$ & $8.3 \pm 0.7^{c}$ & $8.3 \pm 0.7^{c}$ & $10 \pm 0.7^{d}$ & $11 \pm 0.3^{c}$ & $10 \pm 0.6^{c}$ & $9 \pm 0^{c}$ & $11.7 \pm 0.3^{d}$ \\
\hline \multirow{2}{*}{$\begin{array}{c}\text { Nystatin } \\
\text { No }\end{array}$} & $\mathrm{PO}$ & $8 \pm 0^{c}$ & $7.3 \pm 0.3^{b}$ & $8 \pm 0^{d}$ & $8 \pm 0^{\mathrm{b}}$ & $7.3 \pm 0.3^{\mathrm{b}}$ & $8 \pm 0^{\mathrm{b}}$ & $7.7 \pm 0.3^{\mathrm{b}}$ & $8 \pm 0^{\mathrm{b}}$ & $8.3 \pm 0.3^{b}$ & $8 \pm 0^{b}$ & $7.6 \pm 0.3^{b}$ \\
\hline & Dmso & $5 \pm 0^{a}$ & $5 \pm 0^{2}$ & $5 \pm 0^{a}$ & $5 \pm 0^{a}$ & $5 \pm 0^{a}$ & $5 \pm 0^{a}$ & $5 \pm 0^{a}$ & $5 \pm 0^{2}$ & $5 \pm 0^{2}$ & $5 \pm 0^{a}$ & $5 \pm 0^{a}$ \\
\hline
\end{tabular}

Letters $\mathrm{a}, \mathrm{b}, \mathrm{c}, \mathrm{d}, \mathrm{e}, \mathrm{f}$ and $\mathrm{g}$ are distinguishing characters for the significant differences among test fungi assayed with the leaf extracts of Megaphrynium macrostachyum and differentiated based on Duncan test

Table 3. Minimum Inhibitory Concentration (MIC) and Minimum Fungicidal Concentration (MFC) of test extracts on isolated fungi

\begin{tabular}{|c|c|c|c|c|c|c|c|c|}
\hline \multirow{2}{*}{ Isolates } & \multicolumn{2}{|c|}{ Acetone } & \multicolumn{2}{|c|}{ Hexane } & \multicolumn{2}{|c|}{ Ethanol } & \multicolumn{2}{|c|}{ Aqueous } \\
\hline & MIC & MFC & MIC & MFC & MIC & $\mathrm{MFC}$ & MIC & MFC \\
\hline ANS & 0.5 & 5.0 & 10 & - & 0.1 & 1.0 & 10 & 10 \\
\hline AN & 1.0 & 10 & - & - & 0.1 & 0.5 & 10 & 10 \\
\hline AFS & 0.5 & 10 & - & - & 0.1 & 1.0 & - & - \\
\hline MCR & 5.0 & 10 & - & - & 0.1 & 5.0 & 10 & 10 \\
\hline YST & 0.1 & 5.0 & 0.1 & - & 0.1 & 0.1 & 10 & 10 \\
\hline YSTA & 0.1 & 5.0 & - & - & 0.1 & 0.1 & 10 & 10 \\
\hline YSTB & 0.1 & 0.1 & 10 & - & 0.1 & 0.5 & 10 & 10 \\
\hline FSR & 5.0 & - & - & - & 0.5 & 10 & 10 & - \\
\hline $\mathrm{YB}$ & 0.5 & 5.0 & - & - & 0.1 & 1.0 & 10 & - \\
\hline PENU & 0.1 & 5.0 & - & - & 0.1 & 5.0 & 10 & 10 \\
\hline TRI & 0.1 & 0.5 & - & - & 0.1 & - & 5 & 10 \\
\hline
\end{tabular}

ANS: Aspergillus aculeatus, AN: Aspergillus niger, AFS: Aspergillus flavus, MCR: Rhizopus stolonifer, YST: Issatchenkia orientalis, YSTA: Meyerozyma guilliermondii, YSTB: Meyerozyma caribbica, FSR: Fusarium stolonifer, YB: Paecilomyces variotti, PENU: Penicillium crustosum and TRI: Trichoderma harzianum

Table 4. The effect of M. macrostachyum leaf extracts on brine shrimps

\begin{tabular}{|c|c|c|c|c|c|c|c|}
\hline \multirow{2}{*}{$\begin{array}{l}\text { Conc. } \\
(\mu \mathrm{g} / \mathrm{ml})\end{array}$} & \multirow[b]{2}{*}{ Total No } & \multicolumn{2}{|c|}{ Control } & \multirow[b]{2}{*}{ Ethanol } & \multirow[b]{2}{*}{ Hexane } & \multirow[b]{2}{*}{ Acetone } & \multirow[b]{2}{*}{ Aqueous } \\
\hline & & DMSO & $\begin{array}{l}\text { Saline } \\
\text { water }\end{array}$ & & & & \\
\hline 20 & 10 & 0 & 0 & 1 & 4 & 3 & 0 \\
\hline 40 & 10 & 0 & 0 & 1 & 6 & 6 & 0 \\
\hline 60 & 10 & 0 & 0 & 1 & 4 & 7 & 2 \\
\hline 80 & 10 & 0 & 0 & 3 & 7 & 10 & 0 \\
\hline 100 & 10 & 0 & 0 & 5 & 8 & 10 & 1 \\
\hline LC50 & & & & $107.21 \mu \mathrm{g} / \mathrm{ml}$ & $35.41 \mu \mathrm{g} / \mathrm{ml}$ & $31.50 \mu \mathrm{g} / \mathrm{ml}$ & $379.21 \mu \mathrm{g} / \mathrm{ml}$ \\
\hline
\end{tabular}

DMSO: Dimethyl sulfoxide 
Phytochemical determination of leaf extract of $M$. macrostachyum

The results of screened qualitative and quantitative phytochemical constituents of different extracts of $M$. macrsytachyum leaves are shown in Tables 5 .

The qualitative phytochemical constituents of $M$. macrostachyum as shown in Table 5 revealed the presence of alkaloids and saponins in all the test extracts of $M$. macrostachyum leaves, steroids were present in acetone and aqueous extracts and flavonoids were present in ethanol and aqueous extracts.

The result of quantitative phytochemical constituents of ethanol extract of $M$. macrostachyum leaves which was the most active, revealed that the amount of alkaloids, saponins, and flavonoids quantified were $107.48^{\mathrm{mg}} / 100 \mathrm{~g}, 7.66^{\mathrm{mg}} / 100 \mathrm{~g}$ and $56.92^{\mathrm{mg}} / 100 \mathrm{~g}$ respectively and these were depicted in Table 5 .
Essential oils analysis of fresh and dried leaves of $M$. macrostachyum

Tables $6 \mathrm{a}$ and $6 \mathrm{~b}$ show the percentage composition of essential oils of extracts from the dried and fresh leaf of $M$. macrostachyum respectively. The essential oils of fresh and dried leaves of $M$. macrostachyum revealed Isodecane with highest percentage composition, followed by 3',4'-dihydro2'-(morpholin-4-yl)-5',7'-dinitrospiro[cyclopentane-1,3'quinazoline] and 2-(4-chlorophenyl)-1-cyclohexyl-2piperidinoethanone in dried leaves and Hendecane and (1,3,3-Trimethylnonyl) benzene in fresh leaves.

HPLC determination of the ethanol extract of Megaphrynium macrostachyum leaves

The HPLC chromatogram of extract from the leaf of M. macrostachyum is depicted in Fig. 2 and 61 compounds were revealed by the HPLC fingerprinting of the extracts.

Table 5. Qualitative and quantitative phytochemical determination of the extracts

\begin{tabular}{|c|c|c|c|c|c|}
\hline \multirow{2}{*}{ S/No. } & \multirow{2}{*}{ Compound } & \multicolumn{4}{|c|}{ Megaphrynium macrostachyum } \\
\hline & & Acetone extract & Ethanol extract & Hexane extract & Aqueous extract \\
\hline 1 & Alkaloid & + & + & + & + \\
\hline 2 & Tannins & - & - & - & + \\
\hline 3 & Phlobatannins & - & - & - & - \\
\hline 4 & Saponins & + & + & + & + \\
\hline 5 & Flavonoids & - & + & - & + \\
\hline 6 & Steroids & + & - & - & + \\
\hline 7 & Terpenoids & - & - & - & + \\
\hline 8 & Cardiac glycosides & - & - & - & - \\
\hline \multicolumn{6}{|c|}{ Phytoconstituents $(\mathrm{mg} / 100 \mathrm{~g})$} \\
\hline \multirow{2}{*}{\multicolumn{2}{|c|}{ M. macrostachyum }} & Alkaloids & Tannin & Saponins & Flavonoids \\
\hline & & 107.48 & - & 7.66 & 56.92 \\
\hline
\end{tabular}

Table 6a. Essential oil analysis of dried leaves of M. macrostachyum

\begin{tabular}{|c|c|c|c|c|}
\hline \multirow[b]{2}{*}{ Peak No } & \multicolumn{4}{|c|}{ Dried Leaves (Megaphrynium macrostachyum) } \\
\hline & Plant constituents (Essential oils) & RT & RI & Composition (\%) \\
\hline 1 & (E)-2,3-Epoxyoctan-1-ol & 8.199 & 0 & 1.62 \\
\hline 2 & 2,3,4-Trimethylheptane & 8.702 & 823 & 0.67 \\
\hline 3 & (1,3,3-trimethylnonyl)-Benzene & 8.767 & 0 & 2.95 \\
\hline 4 & 2-(2-oxo-2-phenyl-ethyl)-1,3-dioxolane & 8.827 & 0 & 0.87 \\
\hline 5 & $\{$ tris(Mesityl-gallium) [gallium $(\mathrm{H}) \mathrm{t}$-butyl $]$-tetrakis[t-butylphosphane $\}$ & 8.920 & 0 & 2.07 \\
\hline 6 & 2-(2-oxo-2-phenyl-ethyl)-1,3-dioxolane & 9.103 & 0 & 0.89 \\
\hline 7 & Isodecane & 9.424 & 0 & 10.43 \\
\hline 8 & Heptyl hydride & 9.825 & 0 & 0.56 \\
\hline 9 & 1,2,3 Trimethylbenzene & 9.911 & 0 & 1.78 \\
\hline 10 & 5,9,9-Trimethylspiro[3.5]nona-5,7-dien-1-one & 10.508 & 0 & 1.60 \\
\hline 11 & $\begin{array}{l}\text { [1R-(1.alpha.,2.alpha.,6.alpha.)]3,7,7-Trimethylbicyclo[3.1.1]hept-3- } \\
\text { ene-2-thiol }\end{array}$ & 11.003 & 0 & 0.79 \\
\hline 12 & Hendecane & 11.177 & 0 & 1.89 \\
\hline 13 & 2-keto-butyric-acid & 12.046 & 0 & 1.03 \\
\hline 14 & 3,7-Dimethylnonane & 12.779 & 0 & 2.39 \\
\hline 15 & 4-Ethyl-3-Methyldodecane & 15.640 & 0 & 1.65 \\
\hline 16 & 4-Hydroxycarbonyl-2-oxobutyltriphenylphosphonium bromide & 24.386 & 0 & 1.28 \\
\hline 17 & Ethyl .alpha.-fluoro-4-methyl-cinnamate & 25.194 & 1430 & 0.54 \\
\hline 18 & p-Mentha-6,8-dien-2-one, semicarbazone & 26.033 & 1919 & 0.51 \\
\hline 19 & $\begin{array}{c}\text { 3',4'-dihydro-2'-(morpholin-4-yl)-5',7'-dinitrospiro[cyclopentane-1,3'- } \\
\text { quinazoline }]\end{array}$ & 26.794 & 0 & 6.96 \\
\hline 20 & 2-(4-chlorophenyl)-1-cyclohexyl-2-piperidinoethanone & 26.966 & 0 & 2.70 \\
\hline
\end{tabular}


366

\begin{tabular}{|c|c|c|c|c|}
\hline 21 & $\mathrm{~N}$-[(dimethylamino)(t-butylthio)methylene]-benzamide & 27.350 & 0 & 0.53 \\
\hline 22 & $\begin{array}{l}\text { (E)-1-[(1',1'-dimethylethyl)diphenylsilyl]-2- } \\
\text { (trimethylsilyl)ethylene }\end{array}$ & 28.070 & 0 & 0.81 \\
\hline 23 & $\begin{array}{l}\text { 4,4-Dimethyl-2-oxo-tetrahydrofur-3-yl 2-[Tris(trimethylsilyl)silyl]-4- } \\
\text { phenyl-but-3-enoate }\end{array}$ & 29.817 & 0 & 0.37 \\
\hline 24 & Silane & 30.225 & 0 & 0.76 \\
\hline 25 & 2-(N-methyl-N-acetylamino)-4,4-diphenyl-4-ethylcarbonylbutane & 30.875 & 0 & 0.58 \\
\hline 26 & 4-(Benzoylmethyl)-6-methyl-2H-1,4-benzoxazin-3-one & 31.066 & 2498 & 0.57 \\
\hline 27 & Benzoflex & 34.281 & 0 & 0.89 \\
\hline 28 & 4-[[6-(Ethoxycarbonyl)-trans-5-hexenyl $]$ oxy]-6-methyl-2-pyrone & 35.325 & 0 & 0.61 \\
\hline 29 & N,N-Dimethylmesitylphenylacetamide & 36.234 & 0 & 0.57 \\
\hline 30 & diethyl 1,2,3,4,5,6,7,8-octahydro-2,2-isoquinolinedicarboxylate & 36.875 & 0 & 0.52 \\
\hline
\end{tabular}

Table 6b. GC-MS analysis of fresh leaves of Megaphrynium macrostachyum

\begin{tabular}{|c|c|c|c|c|}
\hline \multirow{2}{*}{ Peak No } & \multicolumn{4}{|c|}{ Fresh leaves (Megaphrynium macrostachyum) } \\
\hline & Plant constituents (Essential oils) & RT & RI & Composition (\%) \\
\hline 1 & 2,6-Dimethyloctane & 8.194 & 887 & 2.16 \\
\hline 2 & 2,3-Dimethyloctane & 8.304 & 0 & 0.91 \\
\hline 3 & 5,6-dimethyl- Undecane & 8.583 & 0 & 0.63 \\
\hline 4 & 2-[(4-Chlorobutyl)oxy]tetrahydro-2H-pyran & 8.665 & 1369 & 0.69 \\
\hline 5 & 2,3,4-Trimethylheptane & 8.712 & 823 & 1.20 \\
\hline 6 & 1,3,5-Trimethylbenzene & 8.776 & 0 & 3.50 \\
\hline 7 & 2-Phenylpropane & 8.834 & 0 & 1.15 \\
\hline 8 & 5-Ethyl-2-methylheptane & 8.906 & 887 & 2.63 \\
\hline 9 & 1-methyl-3-(2-methylpropyl)-Cyclopentane & 9.211 & 0 & 2.37 \\
\hline 10 & Isodecane & 9.441 & 0 & 15.56 \\
\hline 11 & (1-methylpropyl)-Benzene & 9.695 & 0 & 1.70 \\
\hline 12 & 2-Butyloctyl alcohol & 9.770 & 1393 & 1.06 \\
\hline 13 & 4-Methyldecane & 9.834 & 10.51 & 2.69 \\
\hline 14 & Mesitylene & 9.921 & 0 & 2.76 \\
\hline 15 & [2-[(2-ethylhexyl)oxy]ethyl]- Cyclohexane & 10.015 & 0 & 0.73 \\
\hline 16 & 2,6-Dimethylheptane & 10.065 & 0 & 0.57 \\
\hline 17 & 4-Cyclohexyl- Tridecane & 10.132 & 0 & 2.61 \\
\hline 18 & Octadecyl hexanoate & 10.196 & 2574 & 0.55 \\
\hline 19 & p-Ethylethylbenzene & 10.363 & 1106 & 1.28 \\
\hline 20 & 2-Phenylbutane & 10.427 & 0 & 2.80 \\
\hline 22 & 2-Methyl-decane & 10.577 & 0 & 1.77 \\
\hline 23 & (1,3,3-Trimethylnonyl)benzene & 10.687 & 1738 & 3.70 \\
\hline 24 & 2-chloro-1,1,1-trifluoro-2-tridecene & 10.853 & 0 & 1.07 \\
\hline 25 & 1,2-Dimethyl-3-ethylbenzene & 10.905 & 0 & 1.54 \\
\hline 26 & Isopropyltoluene & 11.009 & 0 & 1.87 \\
\hline 27 & Hendecane & 11.188 & 0 & 6.43 \\
\hline 28 & p-Cumic aldehyde & 11.278 & 1230 & 0.82 \\
\hline 29 & (1,1,4,6,6-Pentamethylheptyl)benzene & 11.360 & 1654 & 0.61 \\
\hline 30 & Shellsol & 11.443 & 0 & 0.56 \\
\hline 31 & delta.-(3)-dodecanol & 11.879 & 0 & 0.64 \\
\hline 32 & (+)-(R)-p-Mentha-1,8-dien-4-ol & 12.143 & 0 & 0.79 \\
\hline 33 & n-Tridecane & 12.332 & 0 & 0.56 \\
\hline 34 & Adakane 12 & 12.787 & 1214 & 1.81 \\
\hline 35 & $\mathrm{n}$-Tetradecane & 15.652 & 1413 & 1.14 \\
\hline 36 & Octadecan & 18.881 & 0 & 0.71 \\
\hline 37 & 13-Tetradece-11-yn-1-ol & 23.936 & 1663 & 0.57 \\
\hline 38 & 1-(2-Hydroxyethoxy)tridecane & 26.850 & 0 & 0.95 \\
\hline 39 & 2-Decyloxyethanol & 27.027 & 0 & 0.60 \\
\hline 40 & bis(2-ethylhexyl) phthalate & 30.186 & 0 & 0.61 \\
\hline
\end{tabular}

RT: Retention Time

RI: Refractive Index

\%: Percentage 


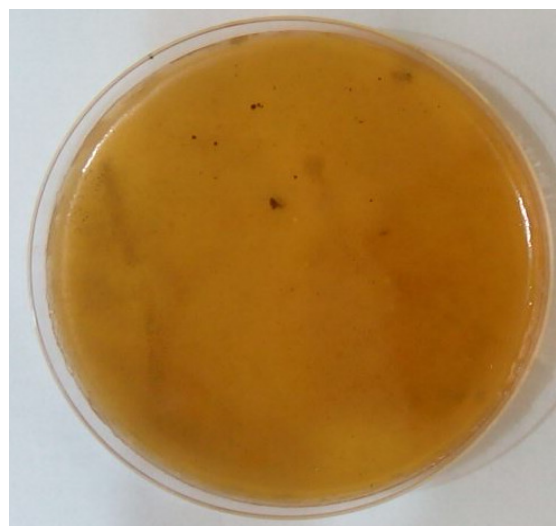

Fig. 1. Purity of the extract on potato dextrose agar

\section{Discussion}

Consumer craving for a healthy diet devoid of risk factor has encouraged the sincere need for natural antimicrobials as a food preservative; this will reduce the employment of heat or synthetic preservatives (Barba et al., 2014). Several researchers have done extensive works on natural antimicrobials from plants as agents of preservation of postharvest crops (Clevelands et al., 2001; Negi, 2012; Palou et al., 2015). This study was aimed at verifying the potency of Megaphrynium macrostachyum as antimicrobial agents against fungi responsible for the spoilage of orange juice and corn Jell-O.

The $M$. macrostachyum leaf extracts from acetone, aqueous, ethanol and hexane were assayed for preservative activity. The percentage yield of the aqueous extract was the highest among the four solvents analyzed and this corroborated earlier work by Do et al. (2014) on the extraction yield of Limnophila aroamtica and this is also in agreement with the works of Dellavallle et al. (2011) and Nurain et al. (2013) that identified aqueous extracts with higher yield compared with other test solvents. Francois et al. (2015) in their study showed that aqueous extracts had higher yield compared with the ethanol, benzene and chloroform extracts and this suggests a higher proportion of water-soluble plant components. The yield of the hexane extracts showed the presence of some nonpolar compounds which support earlier works of Khanum et al. (2014) and Ganatra and Gurubaxani (2016). The considerable yield of the ethanol extract implies that ethanol is a good organic solvent which can help with the release of active phytochemicals adequately (Hwang and Thi, 2004). The production of acetone extracts was quite substantial due to polar and non-polarity status of the solvent which encourages absorption of polar compounds and nonpolar compounds and this assertion uphold earlier reports of Ell of (1998); Sheela et al. (2012); Sivasankaridevi et al. (2013); Dzoyem et al. (2014).

The ascertained pure extract devoid of contamination agrees with the work of Cheesbrough (2000); Okigbo et al. (2009); Anukworji et al. (2012). This study revealed that preservative and fungicidal compounds were present in the leaves of Megaphrynium macrostachyum, since they were able to inhibit the growth of the test fungi, this revelation is in consonance with earlier reports of Grillo and Lawal

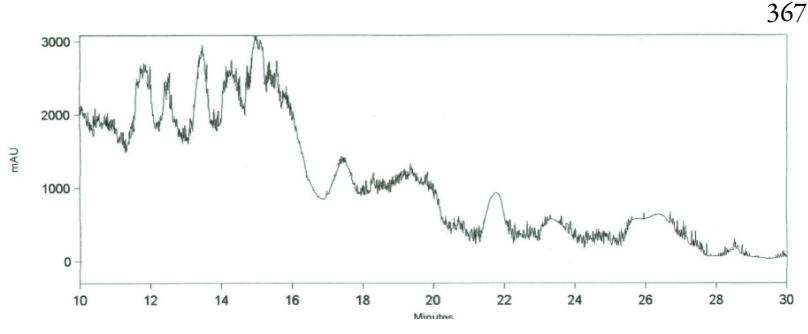

Fig. 2. HPLC Chromatogram of ethanol extract of leaves of $M$. macrostachyum

(2010) and Ajayi and Ojelere (2013) on the bioactivity of the plant on fungal organisms. This study was able to bring to bear the potential of the four extracts in the inhibitory activities against the test fungi. However, the efficacy of the extracts differed based on the concentration of the extract, solvent of extraction and with each test fungus.

The observation of the activities of the extracts against test fungi showed that the activity of the aqueous and hexane extracts against the fungi was quite ineffective at any concentration except in fringes among test organisms which agrees with Kalidindi et al. (2015) that reported that the effect of aqueous extract of Annona squamosa did not demonstrate any significant inhibition on test organisms used. The work of Oluremi et al. (2010) affirmed earlier study of Kalidindi et al. (2015) with an inferred information that some organisms are not susceptible to the extract's activity and the resistance to antimicrobial agents cannot be eliminated but curtailed since some organisms are intrinsically resistant. The less potency of hexane and aqueous extracts also agrees with earlier works by Abubakar et al. (2010). The absence of activity in the hexane and aqueous extracts might either be due to lack of solubility of the active principles (Parekh and Chanda, 2007; Francois et al., 2015) or presence of insufficient quantities of active components in the crude extracts (Taylor et al., 2001).

The activity of acetone and ethanol extracts was quite effective on all test fungi and these occurred as the concentration of the extract increases, which agrees with Vaghasiya and Chanda (2007), Njume et al. (2011), Valle et al. (2015). Wider zone of inhibition was produced at higher concentration and is reduced as the concentration reduces. Cowan (1999) reported that the ethanolic extracts showed better outcomes as compared to aqueous extract because of the ability to extract organic compounds and this boosts the discharge of a larger quantity of active antimicrobial ingredients. The high zone of inhibition exhibited by acetone extracts as noticed in this study corroborates activity of acetone extracts of Arctotis arctotoides, Breonadia salicina, Combretum molle and Gasteria bicolor on some fungal species (Asres et al., 2006; Otang et al., 2012; Mahlo and Eloff, 2014). The wide spectrum of the activity of ethanol extracts on the test isolates might not be unconnected with the solubility of the plant components in the ethanol solvents; more inhibiting effect observed with ethanol extracts than the other extracts could be explained by the fact that ethanol is acidic and in solution donates a proton which makes the medium acidic (Nwankwo et al., 2013; Zhang et al., 2013), any organism in the solution will accept the proton as a base (Nwankwo et al., 2013). The 
368

increase in the concentration of the hydrogen ions inhibits the activities of the microorganism thereby resulting in their death (Uruquiaga and Leighton, 2000). The noticeable reduction in the range of activity in other extracts may portend a possible loss of activity in the event of extraction and further purification of the plant components.

The level of effectiveness of the test extracts based on concentration required to determine the administrative gauze of the extracts on test isolates was established through minimum inhibitory and fungicidal concentrations. The minimum inhibitory and fungicidal concentrations are a test of in-vitro susceptibility that is predictive of in-vivo therapeutic efficacy. The minimum inhibitory concentration which is the lowest concentration of potency of the extract that inhibited the visible growth of fungi after incubation as shown in Table 3 showed acetone and ethanol extracts at $0.1 \mathrm{mg} / \mathrm{ml}$ against Issatchenkia orientalis, Meyerozyma caribbica, Meyerozyma guilliermondii, Penicillium crustossum and Trichoderma harzianum among test fungi with lowest minimum inhibitory concentration needed for the inhibition. The minimum inhibitory concentration in hexane and water were low, observed only with Issatchenkia orientalis when their development was stymied with the hexane extract. The minimum inhibitory concentration activity of the extracts against the test fungi agrees with earlier works on several plants'extracts with acetone and ethanol as solvents of maceration (Erturk, 2006; Awouafack et al., 2013; Santana et al., 2015). The minimum fungicidal concentration which is the concentration of the extracts with no observable growth of fungi after incubation presage that acetone and ethanol extracts of the leaves of $M$. macrostachyum had a low minimum fungicidal concentration on Aspergillus niger, Issatchenkia orientalis, Meyerozyma caribbica, Meyerozyma guilliermondii and Trichoderma harzianum at $\leq 0.5 \mathrm{mg} / \mathrm{ml}$. The minimum fungicidal concentration of hexane and aqueous extracts was quite miniscule, observed only with Issatchenkia orientalis when their growth was hindered with hexane extract. The minimum fungicidal concentration activity of the extracts against the test fungi corroborate several studies earlier conducted by several researchers (Dellavalle et al., 2010; Neela et al., 2014; Rawal et al, 2015; Negi and Chturvedi, 2016).

The in-vivo lethality in brine shrimp test (BST) is a simple and inexpensive test use to evaluate the bioactivity of plant extracts on brine shrimps. The expected response through an assay is lethality and the only available criterion in evaluating is either for the brine shrimp to die or be alive (Pimentel-Montanher et al., 2002; Olorunnisola et al., 2011; Ramachandran et al., 2011). This study showed that the extracts of $M$. macrostachyum from acetone, ethanol, hexane and aqueous displayed significant lethality and nonlethality against brine shrimp nauplii with $\mathrm{LC}_{50}$ of 31.50 $\mu \mathrm{g} / \mathrm{ml}, 107.21 \mu \mathrm{g} / \mathrm{ml}, 35.41 \mu \mathrm{g} / \mathrm{ml}$ and $379.21 \mu \mathrm{g} / \mathrm{ml}$ respectively. The acceptable requirement for general toxicity test for $\mathrm{LC}_{50}$ to kill $50 \%$ of the population of brine shrimp above $100.00 \mu \mathrm{g} / \mathrm{ml}$ is accepted to be nontoxic while below $100.00 \mu \mathrm{g} / \mathrm{ml}$ is indicative of toxicity. The significant lethality and non-lethality of the extracts of $M$. macrostachyum are indications that there is the presence of cytotoxic compounds and also less harmful compounds in the extracts which can be ascribed to the solvents used in extraction.

The richness of plants with phytochemical compounds seemed to be responsible for their bioactivity against microbial organisms. The presence of these bioactive compounds as observed in this study conferred preservative property on the leaf extract of $M$. macrostachyum hence the activity of plant extracts can be ascribed to the presence of antifungal agents that hinder the growth of the test fungi (Martins et al, 2011; Gogoi et al., 2016). The qualitative phytochemical screening of the extracts from the leaf of $M$. macrostachyum revealed the presence of alkaloids, tannins, saponins, flavonoids, steroids and terpenoids. The quantitative phytochemical analysis of the ethanol extracts of $M$. macrostachyum leaves revealed that there were high yields of alkaloids, flavonoids and saponins from the ethanol extracts from leaf of $M$. macrostachyum. The potency in the activeness of the constituents in the plant extracts justified the claims by several scientists that have identified the role of phytochemicals in the inhibition of fungi responsible for spoilage of food (Dellavalle et al., 2011; Cabral et al., 2013; Rakholiya et al., 2014; Negi and Chaturvedi, 2016). The analyzed compounds via GC/MS and HPLC fingerprinting showed constituents in the plants at its crude level and these might be responsible for the inhibition of test isolates. It was mentioned that the complex nature of the phytochemical constituents might be responsible for varying level of activity on fungi isolates and grueling that is required in the determination of process for the target components that is responsible for the fungicidal activity (Balakumar et al., 2011; Ignacimuthu et al., 2011; Stevic et al., 2014; Oro et al., 2015). The fungicidal activity of the extracts might be because of the synergy or antagonistic activities between various elements in the plant extracts (Silva et al, 2010; Balouiri et al., 2016; Salah-Fatnassi et al., 2016).

\section{Conclusions}

The in-vitro evaluation of the plant extracts on the test fungi and the constituents in plant compounds present in the plants showed good inhibition on isolated fungi and indicate the potential of the plants as a preservative agent. The study further portends that the purification and isolation of compounds responsible for inhibition of the test fungi via bio-monitoring will provide us the opportunity of having natural compounds that are safe and without knockon effect on the health status of humans compared to established synthetic preservatives that have been documented to have a repercussive effect.

\section{Acknowledgements}

The research received no specific grant from any funding agency in the public, commercial, or not-for-profit sector.

\section{References}

Abubakar EM (2010). Antibacterial Potential of Crude Leaf Extracts of Eucalyptus camaldulensis against some Pathogenic Fungi. African Journal of PlantScience 4(6):202-209. 
Adegunloye D, Agarry O, Adebolu T, Adetuyi F (2006). Effect of LeafPackaging on the Microbiological assessment of some food Items. African Journal of Biotechnology 5(5):445-447.

Al-Hindi R, Al-Najada A, Mohamed S (2011). Isolation and identification of some fruit spoilage fungi: Screening of plant cell wall degrading enzymes. African Journal of Microbiology Research 5:443-448.

Anukworji C, Puthetti R, Okigbo R(2012). Isolation of Fungi causing rot of Cocoyam (Colocasia esculenta (L.) Schott) and control with Plant Extracts: (Allium sativum, L., Garcinia kola, Heckel, Azadirachta indica L. and Carica papaya, L.). Global Advanced Research Journal of Agricultural Science 1(12):33-47.

Asres, K, Mazumder A, Bucar F (2006). Antibacterial and antifungal activities of extracts of Combretum molle. Ethiopian Medical Journal 44 (3):269-277.

Awouafack M, McGaw L, Gottfried S, Mbouangouere R, Tane P, Spiteller M, EloffJ (2013). Antimicrobial activity and cytotoxicity of the ethanol extract, fractions and eight compounds isolated from Eriosema robustum (Fabaceae). BMC Complementary and Alternative Medicine 13:289296.

Balakumar S, Rajan S, Thirunalasundari T, Jeeva S (2011). Antifungal activity of Ocimum sanctum Linn (Limiaceae) on clinically isolated dermatophytic fungi. Asia Pacific Journal of Tropical Biomedicine 4(8):654-657.

Balouiri M, Sadiki M, Ibnsouda S (2016). Methods for in-vitro evaluating antimicrobial activity: A review. Journal of Pharmaceutical Analysis 6:71-79.

Barba F, Criado M, Belda-Galbis C, Esteve M, Rodrigo D (2015). Stevia rebaudiana Bertoni as a natural antioxidants/antimicrobial for high pressure processed fruit extract: Processing parameter optimization. Food Chemistry 148:126-267.

Borbosa N, Rall L, Fernandes P, Probst I, Fernandes A (2009). Essential oils against foodborne pathogens and spoilage bacteria in minced meat. Foodborne Pathogens and Disease 6(6):725-728.

Cabral L, Pinto V, Patriarca A (2013). Application of plant derived compounds to control fungal spoilage and mycotoxin production in foods. International Journal of Food Microbiology 166:1-14.

Cheesbrough M (2000). District laboratory practice in tropical countries. E.C.B.S. Cambridge University Press, London.

Cleveland J, Montville T, Nes I, Chikinwa M (2001). Bacteriocins: Safe, natural antimicrobial for food preservation. International Journal of Food Microbiology 71:1-20.

Cock I, Van Vuuren S (2015). South African food and medicinal plant extracts as potential antimicrobial food agents. Journal of Food Science and Technology 52(11):6879-6899.

Dellavalle P, Cabrera A, Alem D, Larranaga P, Ferreira F, Rizza M (2011). Antifungal activity of medicinal plant extracts against Phytopathogenic fungus Alternaria spp. Chilean Journal of Agricultural Research 71 (2):231-239.

Do Q, Angkawyaja A, Tran-Nguyen P, Huynh L, Soetaredjo F, IsmadjiS, Ju Y (2014). Effect of extraction solvent on total phenol content, total flavonoid content, and antioxidant activity of Limnophila aromatica. Journal of food and Drugs Analysis 22:296-302.

Dzoyem JP, McGaw LJ, EllofJN (2014). In-vitro antibacterial, antioxidants and cytotoxic activity of acetone leaf extracts of nine under-investigated Fabaceae tree species leads to potentially useful extracts in animal health and productivity. BMC Complementary and Alternative Medicine 14:147-153.

Ellof JA (1998). Sensitive and quick microplate method to determine the minimum inhibitory concentration of plant extracts for bacteria. Planta Medica64:711-713.

Erturk O (2006). Antibacterial and antifungal activity of ethanolic extracts from eleven spice plants. Biological Bratislava 61(3):275-278.

Ganatra S, Gurubaxani S (2016). Preliminary Ppytochemical and TLC profiling of Lantana camara leaf extracts. Journal of Chemical and Pharmaceutical Research 8(15):614-617.

Gogoi J, Nakhuru K, Policegoudra R, Chattopadhyah P, Rai A, Veer V (2016). Isolation and characterization of bioactive components from Mirabilis jalapa L. radix. Journal of Traditional and Complementary Medicine 6:41-47.

Hwang E, Thi N (2014). Effects of extraction and processing methods on antioxidant compound contents and radical scavenging activities of leaves (Porphyra tenera). Preventive Nutrition and Food Science 19(1):40-48.

Ignacimuthu S, Duraipardiyan V (2011). Antifungal activity of traditional medicinal plants from Tamil Nadu, India. Asia Pacific Journal of Tropical BiomedicineS204S215.

Kalidindi N, Thimmaiah N, Jagadeesh N, Nandeep R, Swetha S, Kalidindi $B$ (2015). Antifungal and antioxidant activities of organic and aqueous extracts of Annona squamosa Linn. leaves. Journal of Food and Drug Analysis 23:795-802.

Khanum R, Mazhar F, Johangir M (2014). Antioxidant evaluations of polar and non-polar fractions of Cajanus Cajan seeds. Journal of Medicinal Plants Research 9(6):193-198.

Krasniesioka K, Gniewoez M, Synowiec A, Przybyl J, Baczek K, Weglaiz Z (2014). The use of pullulan coating enriched with plant extracts from Satureja horteneis L. to maintain pepper and apple quality and safety. Postharvest Biology and Technology 90:63-67.

Mahlo S, Eloff J (2014). Acetone leaf extracts of Breonadia salicina (Rubiaceae) and ursolic acid protect oranges against infection by Penicillium species. South African Journal of Botany 93:43-53.

Martins S, Mussatto S, Martinez-Avila G, Montarez-Saenz J, Aguilar C, Teixeira J (2011). Bioactive phenolic compounds: Production and extraction by solid state fermentation. A review Biotechnology Advance 29:365-375.

Maswada H, Abdallah S (2013). In-vitro antifungal activity of three geophytic plant extracts against three post-harvest pathogenic fungi. Pakistan Journal of Biological Science 16(23):1698-1705.

Minhas F, Yasin Y, Awan Z, Ahmed N (2013). Antimicrobial activities of the leaves and roots of Elaeagnus umbellata Thunb. African Journal of Biotechnology 12:67546760.

Mith H, Dure R, Delcenserie V, Zhiri A, Daube G, Clinquart A (2014). Antimicrobial activities of commercial essential oils and their components against food-borne pathogens and food spoilage bacteria. Food Science and Nutrition 2(4):403-416.

Neela F, Sonia L, Shamsi S (2014). Antifungal activity of selected medicinal plant extract on Fusarium oxysporium Schlechtthe causal agent of 
370

Fusarium Wilt Disease in tomato. American Journal of Plant Science 5:2665-2671.

Negi S (2012). Plant extracts for the control of bacterial growth: efficacy, stability and safety issue for food application. International Journal of Food Microbiology 156:7-17.

Negi K, Chaturvedi P (2016). In-vitro antimicrobial efficacy of Rhynchostegium vagans A. Jaeger (Moss) against commonly occurring pathogenic microbes of India sub-tropics. Asian Pacific Journal of Tropical Disease 6(1):10-14.

Njume C, Afolayan A, Samie A, Ndip R (2011). Inhibitory and bactericidal potential of crude acetone extracts of Combretum molle (Combretaceae) on drug resistant strain of Helicobacter pylori. Journal of Health, population and Nutrition 29(5):438-445.

Nurain A, Noriham A, Zainon M, Wan Saidatul W, Khairusy S (2013). Comparative study of aqueous and ethanolic aromatiic Malaysian herbs extracts using four antioxidant activity assays. International Journal of Agricultural Research 8(2):55-66.

Okigbo R, Anuagasi C, Amadi J, Ukpabi U (2009). Potential inhibitory effects of some African tuberous plant extracts on Escherichia coli, Staphylococcus aureus and Candida albicans. International Journal of Integrative Biology 6(2):91-98.

Olorunnisola O, Bradley G, Afolayan A (2011). Antioxidant properties and cytotoxicity evaluation of methanolic extract of dried and fresh rhizomes of Tulbaghia violacea. African Journal of Pharmacy and Pharmacology 5(22):2490-2497.

Oluremi B, Osungunna M, Omafuna O (2010). Comparative assessment of antibacterial activity of Uvaria chamae parts. African Journal of Microbiology Research 4(13):1391-1394.

Oro D, Heissler A, RossiE, Scapin D, Malheiras P, BoffE(2015). Antifungal activity of natural compounds against Candida species isolated from HIV-positive patients. Asian Pacific Journal of Tropical Biomedicine 5(9):781-784.

Otang W, Grierson D, Ndip R (2012). Antifungal activity of Arctotis arctotoides (L. F) O. Hoffm and Gasteria bicolor Haw against opportunities fungi associated with human immunodeficiency virus/acquired immunodeficiency virus/acquired immunodeficiency syndrome. Pharmacognosy Magazine 8(30):135-140.

Palou L, Valenca-Chamorro S, Peroz - Gago M (2015). Antifungal eible coatings for fresh citrus fruits: A Review 5:962-986.

Pimentel-Montanher A, Pizzolatti M, Costa-Brighente I (2002). An application of the brine shrimp bioassay for general screening of Brazilian medicinal plants. Acta Farmaceutica Bonaerense 21(3):175-178.

Rakholiya, K, Vaghelam P, Rathod T, Chanda S (2014). Comparative study of hydroalcoholic extracts of Momordica charantia L. against foodborne pathogens. Indian Journal of Pharmaceutical Sciences 76(2):148-156.

Ramachandran S, Vamsikrichna M, Gowthami K, Heera B, Dhanaraju M. (2011). Assessment of cytotoxic activity of Agavecantula using Brine Shrimp (Artemia salina) lethality bioassay. Asian Journal of Scientific Research 4(1):90-94.

Rawal P, Adhikari R, Danu K, Tiwari A (2015). Antifungal activity of Acorus calamus against Fusarium oxysporium F. sp. Lycopersici. International Journal of Current Microbiology and Applied Sciences 4(1):710-715.
Salah-Fatrassi K, Hassayoun F, Cheraif I, Khan S, Jannet H, Hammami M, Aouni M, Harzallah-Skhiri F (2016). Chemical composition, antibacterial and antifungal activities of flowerhead and root essential oils of Santolina chamaecyparissus L., growing wild in Tunisia. Saudi Journal of Biological Sciences 24(4):875-882.

Santana D, Da Costa R, Araiyo R, Paula J, Silveira E, Braz-Filho R, Espindola L (2015). Activity of Fabaceae species extracts against fungi and Leishmania: Vatacarpan as a novel potent anti-candida agent. Revista Brasileirade Farmacognosia 25:401-406.

Sheela D, Jeeva S, Shamula R, Packia-Lekshmi C, Brindha R (2012). Antimicrobial activity and phytochemical analysis of Sanseiveria roxburghiana leaf. Asian Journal of Plant Science and Research 2(1):4144.

Silva N, Junior F (2010). Biological properties of medicinal plants. A review of their antimicrobial activity. The Journal of Venomous Animals and Toxins including Tropical Disease 16(3):402-413.

Sivasankaridevi T, Rajan A, Maina C, Suvarna V (2013). Antimicrobial Activity of some important edible Leaf Extracts. Insight Microbiology 3(2):15-18.

Skocibusic, M, Bezic N, Dunkic V (2006). Phytochemical composition and antimicrobial activities of the essential oils from Satureja subspicata Vis growing in Crotia. Food Chemistry 96:20-28.

Stevic T, Beric T, Savikin K, Sokovin M, Godevac D, Dunkic I, Stankovic S (2014). Antifungal activity of selected essential oils against fungi isolated from medicinal plant. Industrial Crop and Products 55:116-122.

Tajkarimi MM, Ibrahim SA, Cliver DO (2010). Antimicrobial herb and spice compounds in food. Food Control 21:1199-1218.

Vaghasiya Y, Chanda S (2007). Screening of methanol and acetone extracts of fourteen Indian medicinal plants for antimicrobial activity. Turkey Journal of Biology 31:243-248.

Valle D, Cabrera E, Puzon J, Rivera W (2016). Antimicrobial activities of methanol, ethanol and supercritical $\mathrm{CO}_{2}$ extracts of Philippine Piper betle $\mathrm{L}$. on clinical isolates of Gram Positive and Gram Negative Bacteria with transferable multiple drug resistance. PLoS One 11(1): e0146349.

Wang Q, Cao Y, Zhou H, Jiang C, Feng Y, Wei S (2015). Effect of postharvest curing treatment of flesh colour and phenolic metabolism in fresh-cut potato products. Food Chemistry 169:246-254.

Yazdani D, Tan Y, Zainal A, Jaganath I (2011). A review on bioactive compounds isolated from plants against plant pathogenic fungi. Journal of Medicinal Plants Research 5(30):6584-6589.

Zhang L, Ravipati S, Koyyalamudi S, Jeong S, Reddy N, Bartlett J, Smith P, Cruiz M, Monterro M, Melguizo A, Jimenez E, Vicenta F (2013). Antifungal and antibacterial activities of ethanol extracts of selected traditional Chinese Medicine Herbs. Asian Pacific Journal of Tropical Medicine 6(9):673-681.

Zhou G, Xu L, Liu Y (2010). Preservation technologies for fresh meat - A review. MeatScience 86:119-128. 